\title{
Dynamics and relaxation in spin nematics
}

\author{
V. G. Bar'yakhtar, ${ }^{1}$ V. I. Butrim, ${ }^{2}$ A. K. Kolezhuk, ${ }^{3,1}$ and B. A. Ivanov ${ }^{1,4}$ \\ ${ }^{1}$ Institute of Magnetism, National Academy of Sciences and Ministry of Education, 03142 Kiev, Ukraine \\ ${ }^{2}$ Taurida National V.I. Vernadsky University, 95007 Simferopol, Ukraine \\ ${ }^{3}$ Institute of High Technologies, Taras Shevchenko National University of Kiev, 03022 Kiev, Ukraine \\ ${ }^{4}$ Radiophysics Department, Taras Shevchenko National University of Kiev, 03022 Kiev, Ukraine
}

(Dated: November 3, 2018)

\begin{abstract}
We study dynamics and relaxation of elementary excitations (magnons) in the spin nematic (quadrupole ordered) phase of $S=1$ magnets. We develop a general phenomenological theory of spin dynamics and relaxation for spin-1 systems. Results of the phenomenological approach are compared to those obtained by microscopic calculations for the specific $S=1$ model with isotropic bilinear and biquadratic exchange interactions. This model exhibits a rich behavior depending on the ratio of bilinear and biquadratic exchange constants, including several points with an enhanced symmetry. It is shown that symmetry plays an important role in relaxation. Particularly, at the $\mathrm{SU}(3)$ ferromagnetic point the magnon damping $\Gamma$ depends on its wavevector $k$ as $\Gamma \propto k^{4}$, while a deviation from the high-symmetry point changes the behavior of the leading term to $\Gamma \propto k^{2}$. We point out a similarity between the behavior of magnon relaxation in spin nematics to that in an isotropic ferromagnet.
\end{abstract}

PACS numbers: $76.20 .+\mathrm{q}, 75.10 . \mathrm{Jm}, 75.40 . \mathrm{Gb}, 75.30 . \mathrm{Ds}$

\section{INTRODUCTION}

Exchange interaction between atomic spins in solids at low temperatures usually leads to magnetic ordering, that entails spontaneous breaking of the time reversal symmetry. A large body of results in physics of magnetism have been obtained in the framework of a phenomenological theory, which describes the state of a magnet at low temperature by a constant length magnetization vector $\boldsymbol{M}$ proportional to the average value of the spin operator $\langle\widehat{\boldsymbol{S}}\rangle$ (or, in the more general case of a magnet with $n$ sublattices, by several sublattice magnetization vectors $\left.M_{\alpha}, \alpha=1,2, \ldots n\right), \underline{1}-\underline{\underline{n}}$ This approach naturally leads to the macroscopic description of the spin dynamics by means of the Landau-Lifshitz equations for the magnetization vector (or sublattice magnetizations).

The assumption that the magnitude of the magnetization vector is conserved, $|\boldsymbol{M}|=$ const, is justified in the case of a well pronounced long-range magnetic ordering with $|\boldsymbol{M}|$ being not much different from its saturation value. In that case longitudinal oscillations that change $|\boldsymbol{M}|$ have much higher energies than transversal modes described by the Landau-Lifshitz equations, and thus the longitudinal modes can be safely neglected in the lowenergy, long-wavelength limit. However, the longitudinal modes may come down and become comparable in energy with the transversal ones close to phase boundaries, where thermal or quantum fluctuations lead to a strong "spin contraction" so that $|\langle\widehat{\boldsymbol{S}}\rangle| \ll S$. In that case there might be other low-energy degrees of freedom as well, for instance, those related to the so-called spin nematic ordering ${ }^{4-7}$ that is described by the deGennes tensor order parameter

$$
Q^{a b}=\frac{S(S+1)}{3} \delta_{a b}-\frac{1}{2}\left\langle S^{a} S^{b}+S^{b} S^{a}\right\rangle
$$

built from quadrupole averages. Although the latter expression makes sense only for $S>\frac{1}{2}$, nematic order is possible in spin- $\frac{1}{2}$ systems as well $;-11$ in that case the $S>\frac{1}{2}$ spin operators $S^{a}$ above are understood as composed from $S=\frac{1}{2}$ spins belonging to different lattice sites. In a usual magnetically ordered state, spin nematic (quadrupolar) order $Q^{a b}$ is trivially present as a "slave" of the primary magnetic (dipolar) order $\boldsymbol{M}$. In contrast to that, in a purely nematic state the average (sublattice) magnetization $\boldsymbol{M}$ is absent even at zero temperature, and the only order is characterized by nontrivial quadrupole averages. The time reversal symmetry remains unbroken in the spin nematic phase.

The static and dynamic properties of spin nematics have attracted much interest of researchers during last two decades $, 8,10-27$ This interest has received a new boost recently, mainly in the context of spinor Bose-Einstein condensates (BECs) 28 Optically trapped ultracold gases provide a unique highly controllable environment opening an exciting route for simulating a wide range of strongly correlated systems including quantum magnets. Ongoing experiments ${ }^{29-35}$ have already managed to reach the regime suitable to study magnetic properties of such systems. Spinor gases are especially interesting because their internal degrees of freedom result in a rich physics, providing an opportunity to study quantum magnets with strong non-Heisenberg interactions.

Spin-1 gas represents the simplest bosonic spinor system. Depending on interparticle interactions ${ }^{36,37}$ determined by the $s$-wave scattering lengths $a_{0,2}$ for the collision channels with the total spin 0 and 2, spin-1 BEC has ferromagnetic ground state for $a_{0}>a_{2}$ (as in ${ }^{87} \mathrm{Rb}$, see Ref. 38), and nematic (polar) ground state for $a_{2}>a_{0}$ (as in ${ }^{23} \mathrm{Na}$, see Ref. 39). For spin-1 atoms loaded in an optical lattice a variety of phases has been predicted. $20,40-47$

In the Mott insulator regime (intersite hopping much 
smaller than the on-site interaction), at low energies spin1 bosons on a lattice can be effectively described by a purely spin model (other degrees of freedom are separated by a large energy gap). At odd fillings, this effective model describes $S=1$ spins. Exchange interaction of those spins, $\stackrel{41.43}{\longrightarrow}$ in addition to the usual Heisenberg exchange terms $\left(\boldsymbol{S}_{i} \cdot \boldsymbol{S}_{\boldsymbol{j}}\right)$, includes strong biquadratic exchange of the type $\left(\boldsymbol{S}_{i} \cdot \boldsymbol{S}_{\boldsymbol{j}}\right)^{2}$. In three-dimensional systems, this biquadratic exchange leads to a long-rangeordered spin-nematic state for $a_{2}>a_{0}$, while the case $a_{0}=a_{2}$ exhibits an enlarged $S U(3)$ symmetry with a highly degenerate ground state $\underline{\underline{48}}$

In this paper we develop a general phenomenological theory of spin dynamics and relaxation in isotropic spin-1 magnets, focusing on the properties of the nematic phase in three dimensions. We compare the results of the phenomenological approach to those obtained by microscopic calculations for the $S=1$ lattice model with isotropic bilinear and biquadratic exchange interactions. Our goal is to demonstrate the role of enhanced symmetry in the relaxation. Particularly, we show that at the $S U(3)$ ferromagnetic point the magnon damping $\Gamma$ depends on its wavevector $k$ as $\Gamma \propto k^{4}$, while a deviation from the highsymmetry point changes this behavior to $\Gamma \propto k^{2}$. Our formalism reveals parallels between the general equations describing spin dynamics and relaxation in spin nematics and those proposed earlier by one of the authors $\underline{49}$ for the dynamics and relaxation of magnetization in ferromagnets.

The structure of the paper is as follows: in Sect. II we describe the phenomenological theory of spin dynamics and relaxation, based on the Onsager relations, as it has been done earlier for ferromagnets $\stackrel{49}{=}$ In Sect. IV we discuss the microscopic calculation of magnon relaxation for the $S=1$ bilinear-biquadratic model, and compare the results of the phenomenological and microscopic approaches. Finally, Sect. $\mathrm{D}$ contains a brief summary.

\section{PHENOMENOLOGICAL THEORY OF SPIN DYNAMICS AND RELAXATION IN $S=1$ MAGNETS}

\section{A. Description of spin-1 states and order parameters}

To describe the dynamics of spin- 1 system, it is convenient to use the formalism of $S U(3)$ coherent states $\underline{\underline{15}}$ The most general pure spin- 1 state $|\psi\rangle_{j}$ at a single given site $j$ is a linear superposition of three basis states $|\sigma\rangle_{j}$ with $S_{j}^{z}|\sigma\rangle_{j}=\sigma|\sigma\rangle_{j}, \sigma=0, \pm 1$. It is convenient to write down this state in a "cartesian" basis of states

$$
|\psi\rangle_{j}=\sum_{a=1,2,3} z_{j a}\left|t_{a}\right\rangle_{j}
$$

where $\left|t_{1}\right\rangle=\frac{1}{\sqrt{2}}(|-1\rangle-|+1\rangle),\left|t_{2}\right\rangle=\frac{i}{\sqrt{2}}(|-1\rangle+|+1\rangle)$, $\left|t_{3}\right\rangle=|0\rangle$, then the object $\boldsymbol{z}_{j}=\left(z_{j 1}, z_{j 2}, z_{j 3}\right)$ transforms as a vector under usual $(\mathrm{SU}(2))$ rotations.
Instead of the three-component complex vector $\boldsymbol{z}$, one can use a different spin- 1 state parametrization 50,51 through the eight-component real vector $\boldsymbol{n}$, defined as follows:

$$
n_{\alpha}=z_{a}^{*} \lambda_{a b}^{\alpha} z_{b},
$$

where $\lambda^{\alpha}, \alpha=1, \ldots 8$ are the Gell-Mann matrices that are hermitean and have the following properties:

$$
\begin{aligned}
& \lambda^{\alpha} \lambda^{\beta}=\frac{2}{3} \delta_{\alpha \beta} \mathbb{1}+\left(d_{\alpha \beta \gamma}+i f_{\alpha \beta \gamma}\right) \lambda^{\gamma}, \\
& \lambda_{a b}^{\alpha} \lambda_{a^{\prime} b^{\prime}}^{\alpha}=2 \delta_{a b^{\prime}} \delta_{a^{\prime} b}-\frac{2}{3} \delta_{a b} \delta_{a^{\prime} b^{\prime}} .
\end{aligned}
$$

Here the tensor of structure constants $f_{\alpha \beta \gamma}$ is totally antisymmetric with respect to the permutation of any pair of indices, while the other tensor $d_{\alpha \beta \gamma}$ is totally symmetric under such operations, see the Appendix.

The octet $\boldsymbol{n}$ transforms according to the adjoint representation of the $\mathrm{SU}(3)$ group under a general unitary $(\mathrm{SU}(3))$ transformation of the triplet of basis spin-1 states. For two octets $\boldsymbol{n}$ and $\boldsymbol{n}^{\prime}$, one can define a scalar product $\left(\boldsymbol{n} \cdot \boldsymbol{n}^{\prime}\right)$, vector crossproduct $\left(\boldsymbol{n} \wedge \boldsymbol{n}^{\prime}\right)$, and symmetric vector product $\left(\boldsymbol{n} * \boldsymbol{n}^{\prime}\right)$ as follows: $\underline{.52}$

$$
\begin{aligned}
\boldsymbol{n} \cdot \boldsymbol{n}^{\prime}=n_{\alpha} n_{\alpha}^{\prime}, & \left(\boldsymbol{n} * \boldsymbol{n}^{\prime}\right)_{\alpha}=d_{\alpha \beta \gamma} n_{\beta} n_{\gamma}^{\prime}, \\
& \left(\boldsymbol{n} \wedge \boldsymbol{n}^{\prime}\right)_{\alpha}=f_{\alpha \beta \gamma} n_{\beta} n_{\gamma}^{\prime} .
\end{aligned}
$$

For any octet $\boldsymbol{n}$, quantities

$$
I_{2}(\boldsymbol{n})=\boldsymbol{n} \cdot \boldsymbol{n}, \quad I_{3}(\boldsymbol{n})=\boldsymbol{n} \cdot(\boldsymbol{n} * \boldsymbol{n})
$$

remain invariant under $\mathrm{SU}(3)$ basis tranformations $\boldsymbol{z} \mapsto$ $U \boldsymbol{z}$, and satisfy the constraint

$$
I_{2}(\boldsymbol{n})^{3} \geq 3 I_{3}(\boldsymbol{n})^{2} .
$$

The density matrix $\widehat{\rho}$ of a single spin can be expressed through the octet $\boldsymbol{n}$ :

$$
\left\langle t_{a}|\rho| t_{b}\right\rangle=\frac{1}{3} \delta_{a b}+\frac{1}{2} n_{\alpha} \lambda_{a b}^{\alpha} .
$$

For a normalized pure state, the condition $\widehat{\rho}^{2}=\widehat{\rho}$ translates into the following constraints on $\boldsymbol{n}$ :

$$
\boldsymbol{n}^{2}=\frac{4}{3}, \quad(\boldsymbol{n} * \boldsymbol{n})=\frac{2}{3} \boldsymbol{n},
$$

i.e., $I_{2}(\boldsymbol{n})=4 / 3$ and $I_{3}(\boldsymbol{n})=8 / 9$, so (6) becomes an equality. One can show that the constraints (8) reduce the dimension of the $\boldsymbol{n}$-space to four 12,50

For a mixed state, Eq. (7) retains sense as it is the most general expression for a $3 \times 3$ matrix with unit trace. The constraints (8) generally do not hold for a mixed state. However, under a unitary rotation $\widehat{\rho}(\boldsymbol{n}) \mapsto U^{\dagger} \widehat{\rho}(\boldsymbol{n}) U=$ $\widehat{\rho}\left(\boldsymbol{n}^{\prime}\right)$ the transformation $\boldsymbol{n} \mapsto \boldsymbol{n}^{\prime}$ is still determined by the adjoint representation of $\mathrm{SU}(3)$, so $I_{2,3}(\boldsymbol{n})=I_{2,3}\left(\boldsymbol{n}^{\prime}\right)$ remain invariant, but are no more fixed at the pure state values (8). The dimension of the $\boldsymbol{n}$-space in the general case of a mixed state (7) is equal to six $\underline{12,50}$ 
The components of the octet $\boldsymbol{n}$ correspond to the following on-site spin averages:

$$
\begin{aligned}
& n_{2}=\left\langle S^{z}\right\rangle, \quad n_{5}=-\left\langle S^{y}\right\rangle, \quad n_{7}=\left\langle S^{x}\right\rangle, \\
& n_{1}=\left\langle S^{x} S^{y}+S^{y} S^{x}\right\rangle, \quad n_{4}=-\left\langle S^{x} S^{z}+S^{z} S^{x}\right\rangle, \\
& n_{6}=\left\langle S^{y} S^{z}+S^{z} S^{y}\right\rangle, \\
& n_{3}=\left\langle\left(S^{x}\right)^{2}-\left(S^{y}\right)^{2}\right\rangle, \quad n_{8}=\sqrt{3}\left(\left\langle\left(S^{z}\right)^{2}\right\rangle-2 / 3\right),
\end{aligned}
$$

which can be split into the magnetization (dipole) part $\boldsymbol{m}$ and nematic (quadrupolar) one $\boldsymbol{d}$,

$$
\begin{aligned}
& \boldsymbol{n}=\boldsymbol{m}+\boldsymbol{d}, \\
& \boldsymbol{m}=\left(n_{7},-n_{5}, n_{2}\right), \quad \boldsymbol{d}=\left(n_{1}, n_{3}, n_{4}, n_{6}, n_{8}\right) .
\end{aligned}
$$

It is easy to see that under time reversal operation $\widehat{T}$, the magnetization $\boldsymbol{m}$ changes sign, while the quadrupolar part $\boldsymbol{d}$ remains invariant:

$$
\widehat{T} \boldsymbol{m}=-\boldsymbol{m}, \quad \widehat{T} \boldsymbol{d}=\boldsymbol{d} .
$$

\section{B. Equations of motion for pure spin $S=1$ states}

The effective Lagrangian of a spin-1 system on a lattice, expressed in terms of the complex unit vector $\boldsymbol{z}$, takes the form

$$
\mathcal{L}=\sum_{j} i\left(\boldsymbol{z}_{j}^{*} \cdot \partial_{t} \boldsymbol{z}_{j}\right)-W\left(\left\{\boldsymbol{z}_{j}^{*}, \boldsymbol{z}_{j}\right\}\right)
$$

where $W$ is the energy that can be also expressed through the octets $\left\{\boldsymbol{n}_{j}\right\}$. We will assume that one can pass to the continuum description just by declaring $\boldsymbol{z}$ (or, alternatively, $\boldsymbol{n})$ a smooth field. Then the Lagrange equations of motion for $z^{*}, z$ take the form

$$
i \frac{\partial \boldsymbol{z}^{*}}{\partial t}=-\frac{\delta W}{\delta \boldsymbol{z}},
$$

where the energy $W\left[\boldsymbol{z}^{*}, \boldsymbol{z}\right]=W[\boldsymbol{n}]$ is now a functional of either the complex vector field $\boldsymbol{z}$ or the real octet field $\boldsymbol{n}$, and $\delta / \delta \boldsymbol{z}$ denotes a variational derivative. Introducing generalized fields

$$
\boldsymbol{H}=-\frac{\delta W}{\delta \boldsymbol{n}}, \quad \boldsymbol{h}=-\frac{\delta W}{\delta \boldsymbol{z}},
$$

one can write

$$
\begin{aligned}
& \frac{\partial n_{\alpha}}{\partial t}=-i\left(h_{a} \lambda_{a b}^{\alpha} z_{b}-\text { c.c }\right), \\
& h_{b}=H_{\alpha} \lambda_{a b}^{\alpha} z_{a}^{*},
\end{aligned}
$$

which finally leads to the equation of motion for $\boldsymbol{n}$ :

$$
\frac{\partial \boldsymbol{n}}{\partial t}=2(\boldsymbol{n} \wedge \boldsymbol{H}) .
$$

It is worth noting that if $W$ depends only on magnetization $\boldsymbol{m}$, (15) reduces to the well-known Landau-Lifshitz equation without dissipation, $\partial \boldsymbol{m} / \partial t=-(\boldsymbol{m} \times \delta W / \delta \boldsymbol{m})$, which describes spin dynamics in ferromagnets. Thus, (15) can be viewed as an extension of the Landau-Lifshitz equation that includes the dynamics of quadrupolar degrees of freedom.

\section{General form of the equations of motion and relaxation terms}

General analysis of dissipative forces in spin-1 nematics can be carried out closely following the approach developed for ferromagnets. 49 Equations of motion can generally be cast in the form of Onsager equations

$$
\frac{\partial n_{\alpha}}{\partial t}=\widehat{\Lambda}_{\alpha \beta}(\boldsymbol{n}) H_{\beta},
$$

where the effective fields $\boldsymbol{H}$, defined in (14), play the role of generalized forces that arise at a deviation from equilibrium, and $\widehat{\Lambda}_{\alpha \beta}$ are the kinetic coefficients that generally shall be understood as operators acting on $\boldsymbol{H}$ (physically this corresponds to taking into account the spatial dispersion).

Since different components of the octet vector $\boldsymbol{n}$ have different properties under time reversal, the Onsager theorem about the symmetry of the kinetic coefficients $\Lambda_{\alpha \beta}(\boldsymbol{n})$ gets slightly modified $\stackrel{53}{=}$ If one introduces the factors $\epsilon_{\alpha}= \pm 1$ describing the symmetry of the $\alpha$-th component of $\boldsymbol{n}$ under time reversal,

$$
\widehat{T} n_{\alpha}=\epsilon_{\alpha} n_{\alpha},
$$

then the kinetic coefficients satisfy the following reciprocity relations:

$$
\widehat{\Lambda}_{\beta \alpha}(\boldsymbol{n})=\epsilon_{\alpha} \epsilon_{\beta} \widehat{\Lambda}_{\alpha \beta}(\widehat{T} \boldsymbol{n}) .
$$

It is easy to see that only the symmetric part $\widehat{\Lambda}_{\alpha \beta}^{(s)}$ of the tensor $\widehat{\Lambda}$ contributes to relaxation. Indeed, the dissipative function $Q$ can be written as

$$
\begin{aligned}
Q & =-\frac{1}{2} \frac{d W}{d t}=\frac{1}{2} \int d \boldsymbol{x} \boldsymbol{H} \cdot \frac{\partial \boldsymbol{n}}{\partial t} \\
& =\frac{1}{2} \int d \boldsymbol{x} H_{\alpha} \widehat{\Lambda}_{\alpha \beta} H_{\beta}=\frac{1}{2} \int d \boldsymbol{x} H_{\alpha} \widehat{\Lambda}_{\alpha \beta}^{(s)} H_{\beta} .
\end{aligned}
$$

The antisymmetric part $\widehat{\Lambda}_{\alpha \beta}^{(a)}$ of the kinetic coefficients tensor makes no contribution into dissipation and thus corresponds to the purely dynamic part of the equations of motion. As we have already established, those equations are given by (15), and thus one obtains

$$
\widehat{\Lambda}_{\alpha \beta}^{(a)}(\boldsymbol{n})=-2 f_{\alpha \beta \gamma} n_{\gamma} .
$$

Under the assumption of weak dispersion, one can expand the symmetric part of $\widehat{\Lambda}$ in spatial derivatives, retaining only up to quadratic terms.

Assuming further that the crystal structure of the magnet has an inversion center, one can ignore the term linear in derivatives, so finally we obtain:

$$
\widehat{\Lambda}_{\alpha \beta}^{(s)}(\boldsymbol{n})=\lambda_{\alpha \beta}(\boldsymbol{n})-\tilde{\lambda}_{\alpha \beta, l l^{\prime}}(\boldsymbol{n}) \frac{\partial^{2}}{\partial x_{l} \partial x_{l^{\prime}}}+\ldots,
$$

where $\lambda_{\alpha \beta}$ and $\widetilde{\lambda}_{\alpha \beta, l l^{\prime}}$ satisfy the reciprocity relations similar to (18) with respect to permutations of $\alpha$ and $\beta$, 
and $\widetilde{\lambda}_{\alpha \beta, l l^{\prime}}$ are symmetric with respect to permutations of $l$ and $l^{\prime}$. The dissipation function in this approximation will contain terms quadratic in the effective fields and their derivatives,

$$
Q=\int d \boldsymbol{x}\left\{\lambda_{\alpha \beta} H_{\alpha} H_{\beta}+\widetilde{\lambda}_{\alpha \beta, l l^{\prime}} \frac{\partial H_{\alpha}}{\partial x_{l}} \frac{\partial H_{\beta}}{\partial x_{l^{\prime}}}\right\},
$$

and equations of motion including relaxation terms acquire the following general form:

$$
\begin{aligned}
\frac{\partial n_{\alpha}}{\partial t} & =2 f_{\alpha \beta \gamma} n_{\beta} H_{\gamma}+R_{\alpha} \\
R_{\alpha} & =\frac{\delta Q}{\delta H_{\alpha}}=\lambda_{\alpha \beta} H_{\beta}-\widetilde{\lambda}_{\alpha \beta, l l^{\prime}} \frac{\partial^{2} H_{\beta}}{\partial x_{l} \partial x_{l^{\prime}}} .
\end{aligned}
$$

Similarly as it is done for usual ferromagnets,,$\frac{1.49}{}$ at finite temperatures the energy $W$ in Eq. (12) has to be understood as the free energy, and the entire framework has to be considered as a phenomenological timedependent Ginzburg-Landau theory, with the parameters of the free energy and the dissipative function being some temperature-dependent constants.

\section{PHENOMENOLOGY APPLIED TO BILINEAR-BIQUADRATIC MODEL}

We apply the general formalism presented above to the bilinear-biquadratic model described by the Hamiltonian

$$
\widehat{\mathcal{H}}=-J_{1} \sum_{\langle i j\rangle} \boldsymbol{S}_{i} \cdot \boldsymbol{S}_{j}-J_{2} \sum_{\langle i j\rangle}\left(\boldsymbol{S}_{i} \cdot \boldsymbol{S}_{j}\right)^{2},
$$

where $\boldsymbol{S}_{i}$ are spin-1 operators at the $i$-th lattice site, and the sums are over nearest neighbors. For simplicity, we assume that the lattice is cubic, and the lattice constant is set to unity.

The above model describes the most general isotropic $(S U(2)$ invariant) exchange interaction between two $S=$ 1 spins. At $J_{1}=J_{2}$ the symmetry of the model is enhanced to $S U(3)$. We consider the nematic region in the vicinity of the ferromagnetic $S U(3)$ point, so it is convenient to set

$$
J_{1}=J(1-\delta), \quad J_{2}=J, \quad J>0, \quad 0<\delta<1 / 2 .
$$

Introducing the continuum field $\boldsymbol{n}=\boldsymbol{m}+\boldsymbol{d}$, one can write down the energy $W=\langle\widehat{\mathcal{H}}\rangle$ as

$$
\begin{aligned}
W & =\int d^{3} x\left\{(Z / 2)\left[-\frac{1}{2} J \boldsymbol{n}^{2}+\delta J \boldsymbol{m}^{2}\right]\right. \\
& \left.+\frac{J}{4}\left[(\nabla \boldsymbol{d})^{2}+(1-2 \delta)(\nabla \boldsymbol{m})^{2}\right]\right\},
\end{aligned}
$$

where $Z=6$ is the lattice coordination number, and $(\nabla \boldsymbol{n})^{2} \equiv \sum_{l}\left(\partial \boldsymbol{n} / \partial x_{l}\right)^{2}$. For pure states, the constraints (8) fix the length of $\boldsymbol{n}$; thus, for $\delta>0$, in the ground state the magnetization $\boldsymbol{m}$ vanishes, which corresponds to a uniaxial spin nematic.
At finite temperature $T$, expression (26) has to be replaced by the free energy, $\mathcal{F}=W-T \mathcal{S}$, where $\mathcal{S}$ is the entropy of the system. Below we use a phenomenological expression for the free energy written in the spirit of Landau's theory of phase transitions in a form of expansion on powers of order parameters. This expression generally can be constructed from all possible invariants of the corresponding symmetry group.

We limit ourselves to the case $\delta<1 / 2$, when local spin correlations are ferromagnetic. For $\delta>1 / 2$ the nearest-neighbor spin-spin correlations change their character to antiferromagnetic, and the effective continuum theory has to be modified ( $\boldsymbol{n}$ has to be split into a uniform and staggered components that become smooth fields in the continuum theory ${ }^{54}$ ). The detailed discussion of this interesting case is going far beyond the scope of this article, and below we will limit ourselves to the case $\delta<1 / 2$, paying main attention to the vicinity of ferromagnetic $S U(3)$ point, where $\delta \ll 1$.

As a consequence of the assumed cubic symmetry of the lattice, the tensor of dissipative constants, that enters relaxation forces depending on derivatives, must be diagonal in its space indices,

$$
\widetilde{\lambda}_{\alpha \beta, l l^{\prime}}=\widetilde{\lambda}_{\alpha \beta} \delta_{l l^{\prime}}
$$

Now let us focus separately on the highly symmetric case $\delta=0$ and then study what happens at deviations from this point.

\section{A. $S U(3)$ symmetric case}

At $\delta=0$ the system is $S U(3)$-symmetric, which dictates that at this point both tensors of dissipative constants are diagonal in the octet indices, $\lambda_{\alpha \beta}=\lambda \delta_{\alpha \beta}$ and $\widetilde{\lambda}_{\alpha \beta}=\widetilde{\lambda} \delta_{\alpha \beta}$. The dissipative force $\boldsymbol{R}$ is thus determined by just two constants $\lambda$ and $\tilde{\lambda}$ :

$$
\boldsymbol{R}=\lambda \boldsymbol{H}-\widetilde{\lambda} \nabla^{2} \boldsymbol{H}
$$

The free energy $\mathcal{F}$ will generally, in addition to the gradient term, contain some function $f\left(I_{2}, I_{3}\right)$ of the $S U(3)$ invariants $I_{2}(\boldsymbol{n})$ and $I_{3}(\boldsymbol{n})$, that will have a minimum at certain equilibrium values of $I_{2}\left(\boldsymbol{n}_{0}\right)=I_{2}^{(0)}$ and $I_{3}\left(\boldsymbol{n}_{0}\right)=I_{3}^{(0)}$. Keeping only the lowest order terms in the expansion of $f\left(I_{2}, I_{3}\right)$ around its minimum as well as in the gradient expansion, we obtain

$$
\begin{aligned}
\mathcal{F} & =\int d^{3} x\left\{\frac{\left(\boldsymbol{n}^{2}-I_{2}^{(0)}\right)^{2}}{4 \chi_{2}}+\frac{\left(\boldsymbol{n} \cdot(\boldsymbol{n} * \boldsymbol{n})-I_{3}^{(0)}\right)^{2}}{6 \chi_{3}}\right. \\
& \left.+\frac{\widetilde{J}}{4}(\nabla \boldsymbol{n})^{2}\right\}
\end{aligned}
$$

where $\chi_{2}$ and $\chi_{3}$ play the role of longitudinal susceptibilities. In the above expression for the free energy, all parameters have to be understood as phenomenological constants that are generally some functions of the 
temperature. At low temperatures $T \ll J$, the temperature dependence of the effective exchange parameter $\widetilde{J}$ is rather weak, so in what follows we assume that $\widetilde{J} \approx J$.

The effective field $\boldsymbol{H}=-\delta \mathcal{F} / \delta \boldsymbol{n}$ takes the form

$$
\begin{aligned}
\boldsymbol{H} & =-\left[\left(\boldsymbol{n}^{2}-I_{2}^{(0)}\right) / \chi_{2}\right] \boldsymbol{n} \\
& -\left[\left(\boldsymbol{n} \cdot(\boldsymbol{n} * \boldsymbol{n})-I_{3}^{(0)}\right) / \chi_{3}\right](\boldsymbol{n} * \boldsymbol{n})+\frac{J}{2} \nabla^{2} \boldsymbol{n},
\end{aligned}
$$

where the first two terms do not contribute to the equation of motion (15) in absence of dissipation (note that $\boldsymbol{n} \wedge(\boldsymbol{n} * \boldsymbol{n})=0)$.

Further, at $\delta=0$ the quantity $\boldsymbol{N}=\int d^{3} x \boldsymbol{n}$ is conserved. As a consequence, the equation of motion for $\boldsymbol{n}$ should have the form of a continuity equation $\partial n_{\alpha} / \partial t+\operatorname{div} \boldsymbol{\Pi}_{\alpha}=0$ and so the dissipative force $\boldsymbol{R}$ must have the form $R_{\alpha}=\partial \Pi_{\alpha b} / \partial x_{b}$. It is clear from (27) and (29) that $\boldsymbol{R}$ can be represented in such a form only if $\lambda=0$. Thus, at the $S U(3)$ symmetric point $\delta=0$ we are left with a single relaxation constant $\widetilde{\lambda}$ :

$$
\boldsymbol{R}=-\widetilde{\lambda} \nabla^{2} \boldsymbol{H}
$$

The above argument is fully similar to the treatment of the exchange approximation in ferromagnets $\underline{49}$

Let us calculate the magnon damping in spin nematic at the $S U(3)$-symmetric point. Without loss of generality, the uniform ground state can be chosen as $\boldsymbol{n}=\boldsymbol{n}_{0}$ with

$$
\boldsymbol{n}_{0}=n_{0}(0,0,0,0,0,0,0,-1),
$$

where $n_{0} \equiv \sqrt{I_{2}^{(0)}}$ is also temperature-dependent. This state corresponds to a uniaxial spin nematic with zero magnetization and the nematic director along the $z$-axis. To visualize it, one may think of an ellipsoid with the same symmetry as for the set of quadrupole averages $\left\langle S_{i} S_{j}+S_{j} S_{i}\right\rangle$. For the above state, such an image is a squeezed ellipsoid of rotation, with the ellipsoid axis being directed along the $z$ axis, and with the thickness $\propto\left\langle S_{z}^{2}\right\rangle$ smaller than the diameter $\propto\left\langle S_{x}^{2}\right\rangle=\left\langle S_{y}^{2}\right\rangle$. At zero temperature $n_{0}=2 / \sqrt{3}$ and thus $\left\langle S_{z}^{2}\right\rangle=0$, i.e., the ellipsoid degenerates into a zero thickness disk. Due to the high symmetry of the system, any other choice of $\boldsymbol{n}_{0}$ that can be obtained from (31) by a general $S U(3)$ rotation would give the same physical results. We have chosen the ground state (31) because it has the simplest form in the octet representation.

We consider small deviations from the ground state, $\boldsymbol{n}=\boldsymbol{n}_{0}+\boldsymbol{\eta}$, linearize equations of motion (23) in $\boldsymbol{\eta}$, and look for eigenmodes in the form $\boldsymbol{\eta}=\widetilde{\boldsymbol{\eta}} e^{i(\boldsymbol{k} \cdot \boldsymbol{x}-\omega t)}$. Then the damping $\Gamma_{k}$ is obtained as the imaginary part of the frequency $\omega_{k}=\Omega_{k}-i \Gamma_{k}$. Since we are interested only in the case of weak inhomogeneities, one can expand $\Omega_{k}$ and $\Gamma_{k}$ in powers of the wave vector $\boldsymbol{k}$ and retain only leading terms.

It should be emphasized that in the present work we focus on the behavior of three-dimensional nematics. In three dimensions, the ordered ground state (31) with spontaneously broken symmetry survives up to a certain temperature $T_{c}$, and from numerical simulations the value of the critical temperature is known ${ }^{55}$ to be sufficiently high: around the $S U(3)$ point $T_{c}$ is roughly equal to the microscopic exchange constant $J$. In lowdimensional systems, thermal or quantum fluctuations can destroy the long-range order, making the above approach inapplicable.

It turns out that the dynamics of the components $\eta_{1}$, $\eta_{2}, \eta_{3}$, and $\eta_{8}$ is purely diffusive: the real parts $\Omega_{k}$ of the corresponding frequencies vanish, and the damping behavior is given by

$$
\begin{aligned}
& \Gamma_{1}=\Gamma_{2}=\Gamma_{3}=\frac{1}{2} \tilde{\lambda} J k^{4}, \\
& \Gamma_{8}=C \widetilde{\lambda} k^{2}+\frac{1}{2} \widetilde{\lambda} J k^{4}, \quad C=n_{0}^{2}\left(\frac{2}{\chi_{2}}+\frac{n_{0}^{2}}{\chi_{3}}\right) .
\end{aligned}
$$

The above modes are longitudinal, they correspond to hydrodynamic relaxation of inhomogeneities in the distribution of $\boldsymbol{n}$ and are not connected to any propagating excitations. Let us briefly discuss their physical meaning. The variable $\eta_{2}=m_{z}$ describes the $z$ component of the magnetization, and two variables $\eta_{1}, \eta_{3}$ correspond to quadrupolar averages built from projections of the spin in the $(x y)$ plane. The dynamics of these variables can be viewed as a change of the spin length coupled with the rotation of the system around the $z$ axis. Such a geometry corresponds to the longitudinal mode found in the same model at $\delta<0$ (i.e., in the ferromagnetic state), where this mode acquires a finite frequency and thus becomes propagating. 56 The last diffusive mode $\eta_{8}$ determines the evolution of the variable $\left\langle m_{z}^{2}\right\rangle$ and describes the relaxation of the de Gennes order parameter to its equilibrium value. In the $S U(3)$-symmetric case there is only inhomogeneous relaxation (all damping constants contain $k$ ). As we shall see below, a deviation from this high symmetry point leads to the appearance of a homogeneous relaxation for quadrupolar degrees of freedom, but not for the total magnetic moment.

The rest of the modes are dynamical and correspond to propagating generalized magnons that unite dipolar (spin) and quadrupolar excitations. The dynamics of $\eta_{4}$ and $\eta_{5}$ is coupled, and the same holds for the pair $\eta_{6}, \eta_{7}$. The corresponding eigenfrequencies are

$$
\begin{aligned}
& \omega_{(45)}= \pm \Omega_{(45)}-i \Gamma_{(45)}, \quad \omega_{(67)}= \pm \Omega_{(67)}-i \Gamma_{(67)},(33) \\
& \Omega_{(45)}=\Omega_{(67)}=\frac{J n_{0} \sqrt{3}}{2} k^{2}, \quad \Gamma_{(45)}=\Gamma_{(67)}=\frac{1}{2} \widetilde{\lambda} J k^{4} .
\end{aligned}
$$

One can see that at small wavevectors the magnon damping behaves as $\Gamma_{k} \propto k^{4}$, while the real part of the frequency $\Omega_{k} \propto k^{2} \gg \Gamma_{k}$, so magnons remain well-defined excitations. 


\section{B. Spin nematic away from the $S U(3)$ point}

Consider a system in the nematic phase $(1 / 2>\delta>0)$. In the ground state the magnetization $\boldsymbol{m}$ vanishes, so the equilibrium value $\boldsymbol{n}=\boldsymbol{n}_{0} \equiv \boldsymbol{d}_{0}$ lies completely within the quadrupolar subspace. The symmetry group is now reduced to $S U(2)$ which is a subgroup of $S U(3)$. Under rotations, $\boldsymbol{m}$ and $\boldsymbol{d}$ transform according to $D^{1}$ and $D^{2}$ representations of the $S U(2)$ group, respectively. Thus, $\boldsymbol{m}^{2}$ and $\boldsymbol{d}^{2}$ are invariants that can separately enter the free energy. The $S U(3)$ invariants $I_{2}(\boldsymbol{n})$ and $I_{3}(\boldsymbol{n})$, of course, remain invariant under any subgroup transformation; since $I_{2}=\boldsymbol{m}^{2}+\boldsymbol{d}^{2}$, we shall only take into account $I_{3}(\boldsymbol{n})$ as another independent invariant. We will assume that the free energy has the form

$$
\begin{aligned}
\mathcal{F} & =\int d^{3} x\left\{\frac{Z}{2} \delta J \boldsymbol{m}^{2}+\frac{\left(\boldsymbol{d}^{2}-\boldsymbol{n}_{0}^{2}\right)^{2}}{4 \chi_{d}}\right. \\
& +\frac{\left[\boldsymbol{n} \cdot(\boldsymbol{n} * \boldsymbol{n})-I_{3}\left(\boldsymbol{n}_{0}\right)\right]^{2}}{6 \chi_{3}} \\
& \left.+\frac{J}{4}\left[(\nabla \boldsymbol{d})^{2}+(1-2 \delta)(\nabla \boldsymbol{m})^{2}\right]\right\},
\end{aligned}
$$

where we have neglected the terms of higher than quadratic order in $\boldsymbol{m}$ and $\left(\boldsymbol{d}-\boldsymbol{n}_{0}\right)$.

Similar to (10), we can divide the effective field $\boldsymbol{H}$ into two components $\boldsymbol{H}_{m}$ and $\boldsymbol{H}_{d}$ lying in the dipolar and quadrupolar subspaces of the octet space, and transforming according to $D^{1}$ and $D^{2}$ representations of the $S U(2)$ group, respectively. Then, the symmetry dictates the following structure of the dissipative constants tensors:

$$
\boldsymbol{R}=0 \cdot \boldsymbol{H}_{m}-\widetilde{\lambda}_{m} \nabla^{2} \boldsymbol{H}_{m}+\lambda_{d} \boldsymbol{H}_{d}-\widetilde{\lambda}_{d} \nabla^{2} \boldsymbol{H}_{d},
$$

where the vanishing constant in front of $\boldsymbol{H}_{m}$ is the consequence of the fact that the total magnetization $\boldsymbol{M}=$ $\int d^{3} x \boldsymbol{m}$ is an integral of motion. From our analysis of the $S U(3)$-symmetric point it also follows that

$$
\lambda_{d} \rightarrow 0, \quad \widetilde{\lambda}_{m} \rightarrow \widetilde{\lambda}_{d} \quad \text { at } \quad \delta \rightarrow 0 .
$$

Eqs. (35536) are valid on both sides of the $S U(3)$ point (i.e., at any sign of $\delta$ ), while $\lambda_{d}$ is strictly non-negative. If one assumes $\lambda_{d}$ to behave analytically as the function of $\delta$, then it follows that

$$
\lambda_{d}=O\left(\delta^{2}\right) \quad \text { at } \quad \delta \rightarrow 0,
$$

however, on general grounds one cannot exclude the possibility of singular behavior of $\lambda_{d}(\delta)$. We will check the above assumption and its consequence (37) in the next section by comparing it with the results of microscopic analysis.

Let us now calculate the magnon damping away from the $S U(3)$ point. Without loss of generality, we assume the nematic ground state with the director along the $z$ axis, i.e., $\boldsymbol{n}_{0}$ of the form (31). We linearize equations of motion in the deviation $\boldsymbol{\eta}=\boldsymbol{n}-\boldsymbol{n}_{0}$ and find the complex eigenfrequencies $\omega=\Omega-i \Gamma$, as we have done before in the $S U(3)$ case. We find that there are again four longitudinal, purely diffusive $(\Omega=0)$, decoupled modes $\eta_{1}, \eta_{2}, \eta_{3}$, and $\eta_{8}$, with the following linewidths:

$$
\begin{aligned}
& \Gamma_{1}=\Gamma_{3}=\frac{1}{2} J k^{2}\left(\lambda_{d}+\widetilde{\lambda}_{d} k^{2}\right), \\
& \Gamma_{2}=\widetilde{\lambda}_{m} J k^{2}\left[Z \delta+\frac{1}{2}(1-2 \delta) k^{2}\right], \\
& \Gamma_{8}=\left(C+\frac{1}{2} J k^{2}\right)\left(\lambda_{d}+\widetilde{\lambda}_{d} k^{2}\right), \quad C=n_{0}^{2}\left(\frac{2}{\chi_{d}}+\frac{n_{0}^{2}}{\chi_{3}}\right) .
\end{aligned}
$$

Comparing the above expressions with their $S U(3)$ counterparts in Eqs. (32), one can see that the main effect of breaking the $S U(3)$ symmetry is the appearance of a homogeneous relaxation in the $\eta_{8}$ quadrupolar order parameter proportional to $\left\langle\left(S^{z}\right)^{2}-2 / 3\right\rangle: k=0$ fluctuations of $\left\langle S_{z}^{2}\right\rangle$ decay with the characteristic relaxation time

$$
\tau_{8} \equiv \frac{1}{\Gamma_{8}(k=0)}=\frac{1}{C \lambda_{d}},
$$

which diverges at $\delta \rightarrow 0$ according to Eq. (37).

As before, there is no homogeneous relaxation of the magnetization, because the $S U(2)$ symmetry remains intact and the total magnetization is conserved. For the other three diffusive modes $\eta_{1,2,3}$, there is a small (of the order of $\delta$ ) splitting between the damping constants of the quadrupolar modes $\left(\eta_{1,3}\right)$ and the magnetization mode $\left(\eta_{2}\right)$, and the leading term in the damping at small wave vectors behaves as $k^{2}$ (in contrast to the $k^{4}$ behavior in the $S U(3)$ case).

The remaining two degenerate eigenmodes correspond to coupled $\left(\eta_{4}, \eta_{5}\right)$ and $\left(\eta_{6}, \eta_{7}\right)$ oscillations, and describe propagating magnons. The corresponding eigenfrequencies $\Omega_{(45)}=\Omega_{(67)}$ and dampings $\Gamma_{(45)}=\Gamma_{(67)}$ are given by

$$
\begin{aligned}
\Omega_{(45)}=\Omega_{(67)} & =\frac{J n_{0} \sqrt{3}}{2}|k|\left(2 Z \delta+(1-2 \delta) k^{2}\right)^{1 / 2}, \\
\Gamma_{(45)}=\Gamma_{(67)} & =\frac{J k^{2}}{4}\left\{\lambda_{d}+2 Z \delta \widetilde{\lambda}_{m}\right. \\
& \left.+\left[\widetilde{\lambda}_{d}+(1-2 \delta) \widetilde{\lambda}_{m}\right] k^{2}\right\} .
\end{aligned}
$$

It is easy to see that away from the $S U(3)$ point magnons acquire linear dispersion, in agreement with previous results obtained by different methods,,$\frac{5,6,15}{1}$ and the leading term in their damping at small wavevectors behaves as $k^{2}$. At $\delta \rightarrow 0$, taking into account (36), we recover our results obtained above for the $S U(3)$ point.

\section{MICROSCOPIC ANALYSIS OF THE BILINEAR-BIQUADRATIC MODEL}

Our goal is to compare the results for magnon damping in the bilinear-biquadratic model (24), obtained above within the phenomenological approach, with a calculation from first principles. We would like to pass to 
the second-quantization formalism for the description of magnons. It is convenient to break up the complex vector $\boldsymbol{z}$, describing the coherent state (10), into two real vectors representing its real and imaginary parts $\boldsymbol{z}=\boldsymbol{u}+i \boldsymbol{v}$. When the normalization condition and the arbitrariness of the phase factor are taken into account, these vectors satisfy the conditions:

$$
\boldsymbol{u}^{2}+\boldsymbol{v}^{2}=1, \quad \boldsymbol{u} \cdot \boldsymbol{v}=0
$$

In terms of the variable vectors $\boldsymbol{u}$ and $\boldsymbol{v}$ the Lagrangian of the system can be written as

$$
\begin{aligned}
\mathcal{L}= & -2 \hbar \sum_{j} \boldsymbol{v}_{j} \partial_{t} \boldsymbol{u}_{j}-W \\
W= & \sum_{\langle i j\rangle}\left\{2\left(J_{2}-J_{1}\right)\left[\left(\boldsymbol{u}_{i} \boldsymbol{u}_{j}\right)\left(\boldsymbol{v}_{i} \boldsymbol{v}_{j}\right)-\left(\boldsymbol{u}_{i} \boldsymbol{v}_{j}\right)\left(\boldsymbol{v}_{i} \boldsymbol{u}_{j}\right)\right]\right. \\
& \left.-\frac{J_{2}}{2}\left[\left(\boldsymbol{u}_{i} \boldsymbol{u}_{j}+\boldsymbol{v}_{i} \boldsymbol{v}_{j}\right)^{2}+\left(\boldsymbol{u}_{i} \boldsymbol{v}_{j}-\boldsymbol{v}_{i} \boldsymbol{u}_{j}\right)^{2}\right]\right\}
\end{aligned}
$$

The spin nematic state, favored at $J_{2}>J_{1}>0$, corresponds to the following condition: vectors $\boldsymbol{u}_{j}$ at all the sites are parallel, $\boldsymbol{u}_{j}=\boldsymbol{u}_{0},\left|\boldsymbol{u}_{0}\right|=1$, and $\boldsymbol{v}_{j}=0$. (There is also an alternative configuration obtained by the substitutions $\boldsymbol{u}_{j} \mapsto \boldsymbol{v}_{j}, \boldsymbol{v}_{j} \mapsto-\boldsymbol{u}_{j}$, but those two states are physically identical).

Consider small deviations of the variables $\boldsymbol{u}$ and $\boldsymbol{v}$ from the spin nematic state. Assume, for the sake of definiteness, that $\boldsymbol{u}_{0}=\boldsymbol{e}_{z}$. Then, according to (41), $v_{z}$ and $u_{z}$ are dependent variables quadratic in the remaining components $u_{x, y}$ and $v_{x, y}$. We can thus expand the Lagrangian in powers of the independent variables $u_{x, y}$ and $v_{x, y}$, keeping terms up to the quartic order. From the kinetic part of the Lagrangian it is obvious that $u_{x}$ and $u_{y}$ can be chosen as the coordinates, while $-2 \hbar v_{x}$ and $-2 \hbar v_{y}$ play the role of the corresponding canonical momenta. It is easy then to pass from the Lagrangian to the Hamiltonian. Passing from variables $\boldsymbol{u}_{j}, \boldsymbol{v}_{j}$ to their Fourier amplitudes $\widetilde{\boldsymbol{u}}_{\boldsymbol{k}}, \widetilde{\boldsymbol{v}}_{\boldsymbol{k}}$ and performing quantization, we can write the standard representation of the coordinates and momenta in terms of the Bose creation and annihilation operators:

$$
\begin{aligned}
& \widetilde{u}_{x, \boldsymbol{k}}=\sqrt{\frac{\hbar}{2 A_{\boldsymbol{k}}}}\left(a_{\boldsymbol{k}}^{\dagger}+a_{-\boldsymbol{k}}\right), \widetilde{v}_{x, \boldsymbol{k}}=i \sqrt{\frac{A_{\boldsymbol{k}}}{8 \hbar}}\left(a_{\boldsymbol{k}}^{\dagger}-a_{-\boldsymbol{k}}\right), \\
& \widetilde{u}_{y, \boldsymbol{k}}=\sqrt{\frac{\hbar}{2 A_{\boldsymbol{k}}}}\left(b_{\boldsymbol{k}}^{\dagger}+b_{-\boldsymbol{k}}\right), \widetilde{v}_{y, \boldsymbol{k}}=i \sqrt{\frac{A_{\boldsymbol{k}}}{8 \hbar}}\left(b_{\boldsymbol{k}}^{\dagger}-b_{-\boldsymbol{k}}\right),(4
\end{aligned}
$$

where $A_{\boldsymbol{k}}=2 \hbar \sqrt{\alpha_{\boldsymbol{k}} / \beta_{\boldsymbol{k}}}$, and we use the notation

$$
\begin{array}{r}
\alpha_{\boldsymbol{k}}=Z J_{2}[1-\gamma(\boldsymbol{k})], \quad \beta_{\boldsymbol{k}}=J_{2} k_{0}^{2} \gamma(\boldsymbol{k})+\alpha_{\boldsymbol{k}}, \\
k_{0}^{2}=2 Z\left(1-J_{1} / J_{2}\right) \equiv 2 Z \delta, \quad \gamma(\boldsymbol{k})=\frac{1}{Z} \sum_{\boldsymbol{\ell}} e^{i \boldsymbol{k} \ell}
\end{array}
$$

$\ell$ being the set of vectors connecting a site of the lattice to its nearest neighbors.
The Hamiltonian decomposes into a sum of quadratic and quartic terms: $\widehat{H}=\widehat{H}_{2}+\widehat{H}_{4}$. The quadratic part $\mathrm{H}_{2}$ becomes diagonal,

$$
H_{2}=\sum_{k} \varepsilon_{\boldsymbol{k}}\left(a_{\boldsymbol{k}}^{\dagger} a_{\boldsymbol{k}}+b_{\boldsymbol{k}}^{\dagger} b_{\boldsymbol{k}}\right)
$$

and the magnon dispersion relation reads

$$
\varepsilon_{\boldsymbol{k}}=\sqrt{\alpha_{\boldsymbol{k}} \beta_{\boldsymbol{k}}}
$$

The spectrum contains two degenerate magnon modes with orthogonal polarizations, that are gapless at $k \rightarrow 0$ in accordance to the Goldstone theorem. For $J_{1}>J_{2}$ this spectrum becomes unstable, which corresponds to a transition to the ferromagnetic state.

In this paper, we are interested in the low-energy dynamics. In the vicinity of the ferronematic $S U(3)$ point $J_{1}=J_{2}$ (i.e., $\delta=0$ ) this is translated into the long-wavelength approximation $k \ll 1$, so we obtain $\gamma(\boldsymbol{k}) \approx 1-k^{2} / Z$ and

$$
\varepsilon_{\boldsymbol{k}}=J_{2} k \sqrt{k_{0}^{2}+k^{2}} \quad \text { at } k \ll 1
$$

The quartic Hamiltonian $\widehat{H}_{4}$ is rather cumbersome, but for our purpose of calculating the magnon damping in the lowest order of the perturbation theory it can be simplified substantially. Indeed, the analysis shows that decay processes (one magnon decaying into three) give a small contribution at low energies, see the note after Eq. (52) below. Thus, the main contribution to the damping comes from terms containing an equal number of creation and annihilation operators. When only such terms are taken into account, the Hamiltonian takes the form

$$
\begin{aligned}
\widehat{H}_{4} & =\frac{1}{N} \sum_{1,2,3,4} \Delta_{1+2-3-4}\left\{\Phi a_{1}^{\dagger} a_{2}^{\dagger} b_{3} b_{4}+\Psi a_{1}^{\dagger} b_{2}^{\dagger} a_{3} b_{4}\right. \\
& \left.+F\left(a_{1}^{\dagger} a_{2}^{\dagger} a_{3} a_{4}+b_{1}^{\dagger} b_{2}^{\dagger} b_{3} b_{4}\right)+\text { h.c. }\right\}
\end{aligned}
$$

where for the sake of brevity we use the shorthand notation $1 \equiv \boldsymbol{k}_{1}$, etc.

The amplitudes $\Phi, \Psi$, and $F$ in Eq. (48) depend on all four magnon momenta. $\Phi$ corresponds to processes of conversion between pairs of magnons with different polarizations, while the other two amplitudes describe magnon scattering. Further, in our case (in the vicinity of the ferronematic $S U(3)$ point) for low-energy processes the momenta of all the magnons participating in the process are small, and one can use the long-wavelength asymptotic expressions for the corresponding amplitudes: 


$$
\begin{aligned}
\Phi_{1234}= & \frac{J_{2}^{3}}{32 \sqrt{\varepsilon_{1} \varepsilon_{2} \varepsilon_{3} \varepsilon_{4}}}\left\{-k_{0}^{2}\left(4 k_{1} k_{2} k_{3} k_{4}+\mu_{1,2}^{+} \mu_{3,4}^{+}\right)+\left(\boldsymbol{k}_{1} \boldsymbol{k}_{3}+\boldsymbol{k}_{2} \boldsymbol{k}_{4}\right)\left(\eta_{1,2}^{-} \eta_{3,4}^{-}-\mu_{1,2}^{-} \mu_{3,4}^{-}\right)\right. \\
& \left.+\left(\boldsymbol{k}_{1} \boldsymbol{k}_{4}+\boldsymbol{k}_{2} \boldsymbol{k}_{3}\right)\left(\eta_{1,2}^{-} \eta_{3,4}^{-}+\mu_{1,2}^{-} \mu_{3,4}^{-}\right)\right\} \\
\Psi_{1234}= & \frac{J_{2}^{3}}{16 \sqrt{\varepsilon_{1} \varepsilon_{2} \varepsilon_{3} \varepsilon_{4}}}\left\{k_{0}^{2}\left(\mu_{1,3}^{-} \mu_{2,4}^{-}-4 k_{1} k_{2} k_{3} k_{4}\right)-\left(\boldsymbol{k}_{1} \boldsymbol{k}_{2}+\boldsymbol{k}_{3} \boldsymbol{k}_{4}\right)\left(\eta_{1,3}^{+} \eta_{2,4}^{+}+\mu_{1,3}^{+} \mu_{2,4}^{+}\right)\right. \\
+ & \left.\left(\boldsymbol{k}_{1} \boldsymbol{k}_{4}+\boldsymbol{k}_{2} \boldsymbol{k}_{3}\right)\left(\eta_{1,3}^{+} \eta_{2,4}^{+}-\mu_{1,3}^{+} \mu_{2,4}^{+}\right)\right\} \\
F_{1234}= & \frac{J_{2}^{3}}{32 \sqrt{\varepsilon_{1} \varepsilon_{2} \varepsilon_{3} \varepsilon_{4}}}\left\{k_{0}^{2}\left(4 k_{1} k_{2} k_{3} k_{4}+k_{1} k_{2} \eta_{3,4}^{+}+k_{3} k_{4} \eta_{1,2}^{+}-\mu_{1,2}^{+} \mu_{3,4}^{+}\right)+\frac{k^{2}}{2}\left(\eta_{1,2}^{-} \eta_{3,4}^{-}+\mu_{1,2}^{-} \mu_{3,4}^{-}\right)\right. \\
- & \left.2\left(\boldsymbol{k}_{1} \boldsymbol{k}_{2}+\boldsymbol{k}_{3} \boldsymbol{k}_{4}\right) \mu_{1,3}^{+} \mu_{2,4}^{+}-2\left(\boldsymbol{k}_{1} \boldsymbol{k}_{3}+\boldsymbol{k}_{2} \boldsymbol{k}_{4}\right) \mu_{1,2}^{-} \mu_{3,4}^{-}\right\} .
\end{aligned}
$$

Here we have used the shorthand notation

$$
\begin{aligned}
& \eta_{n, m}^{ \pm}=k_{n} k_{m} \pm \delta_{n} \delta_{m}, \quad \mu_{n, m}^{ \pm}=k_{n} \delta_{m} \pm \delta_{n} k_{m} \\
& \delta_{n}=\sqrt{k_{0}^{2}+k_{n}^{2}}, \quad k^{2}=k_{1}^{2}+k_{2}^{2}+k_{3}^{2}+k_{4}^{2} .
\end{aligned}
$$

The magnon damping $\Gamma$ is determined as the imaginary part of the self-energy calculated in the second order of perturbation theory. The expression for the damping can be written in the following form:

$$
\begin{aligned}
\Gamma_{\boldsymbol{k}, T} & =\frac{\pi}{2 N^{2}} \sinh \left(\frac{\varepsilon_{\boldsymbol{k}}}{2 T}\right) \sum_{\boldsymbol{p}, \boldsymbol{q}}\left(\Phi^{2}+2 \Psi^{2}+4 F^{2}\right) \\
& \times \frac{\delta\left(\varepsilon_{\boldsymbol{k}}+\varepsilon_{\boldsymbol{p}}-\varepsilon_{\boldsymbol{q}}-\varepsilon_{\boldsymbol{k}+\boldsymbol{p}-\boldsymbol{q}}\right)}{\sinh \left(\frac{\varepsilon_{\boldsymbol{p}}}{2 T}\right) \sinh \left(\frac{\varepsilon_{\boldsymbol{q}}}{2 T}\right) \sinh \left(\frac{\varepsilon_{\boldsymbol{k}+\boldsymbol{p}-\boldsymbol{q}}}{2 T}\right)},
\end{aligned}
$$

where $T$ is the temperature in energy units, and the arguments of the amplitudes (49) are taken as $\boldsymbol{k}_{1}=\boldsymbol{k}$, $\boldsymbol{k}_{2}=\boldsymbol{p}, \boldsymbol{k}_{3}=\boldsymbol{q}, \boldsymbol{k}_{\mathbf{4}}=\boldsymbol{k}+\boldsymbol{p}-\boldsymbol{q}$. We will consider the case of low excitation energies and temperatures well below the critical temperature $T_{c} \sim J_{2}$,

$$
\varepsilon_{\boldsymbol{k}} \ll T \ll J_{2},
$$

then the main contribution to the damping comes from thermally excited magnons with wave vectors $p$ much higher than the magnon wave vector $k$. This justifies neglecting magnon decays in the interaction Hamiltonian: although such processes are allowed by the energy and momentum conservation, their phase volume is proportional to $k^{2}$ and thus their contribution is small.

We will also assume that we are not too far away from the $S U(3)$ point, so that

$$
T \gg J_{2} k_{0}^{2}=2 Z\left(J_{2}-J_{1}\right) .
$$

At the $S U(3)$ point $\left(k_{0}=0, \delta_{n}=k_{n}\right)$ the amplitude $\Phi$ vanishes, and in the immediate vicinity of the $S U(3)$ point determined by (53) it is much smaller than the other two amplitudes, so $\Phi$ can be neglected in the calculation of the damping.

Then, in the leading order in $\left(J_{2}-J_{1}\right)$ and $k / p$, one can drop $k_{0}$ in the dispersions of thermal magnons, and the amplitudes in (51) can be replaced by

$$
\Psi_{1234}=2 F_{1234}=-\frac{J_{2}^{3 / 2}}{4 \sqrt{\varepsilon_{1}}}\left(k_{1}+\delta_{1}\right)\left(\boldsymbol{k}_{1} \cdot \boldsymbol{k}_{2}+\boldsymbol{k}_{3} \cdot \boldsymbol{k}_{4}\right),
$$

where we have dropped contributions proportional to $k_{0}^{2} / k_{1} k_{2} \ll 1$. Further, if $k \gg k_{0}$, then $k_{0}$ can be neglected in the equation for the mass surface as well, and then the result for the damping under the assumptions (52), (53) can be easily obtained as follows:

$$
\Gamma_{k, T}=\frac{J_{2}}{4} C_{k, T} k^{2}\left(2 k^{2}+k_{0}^{2}+O\left(k_{0}^{4}\right)\right) \quad \text { at } \quad k_{0}^{2} \ll k^{2},
$$

where $C_{k, T}$ is a dimensionless factor that weakly (logarithmically) depends on the wave vector $k$ :

$$
C_{k, T}=\frac{T^{2}}{8 \pi^{3} J_{2}^{2}}\left\{\left(\ln \frac{T}{\varepsilon_{k}}+\frac{5}{3}\right)^{2}-\frac{4}{9}-(1-\ln 2)^{2}\right\} .
$$

At $\delta=0$ this result reduces to the one found before for the $S U(3)$ symmetric case $\stackrel{22}{2}$ The appearance of the logarithm above is similar to the well known result for isotropic ferromagnets $\frac{57}{t}$

Comparing (55) to the phenomenological result (40), one can see that it is possible to match those two expressions if one sets the correspondence

$$
\begin{aligned}
& \tilde{\lambda}_{m} \approx \tilde{\lambda}_{d} \mapsto C_{k, T}, \quad \lambda_{d}=O\left(\left(J_{2}-J_{1}\right)^{2}\right), \\
& \tilde{\lambda}_{m}-\widetilde{\lambda}_{d}=O\left(J_{2}-J_{1}\right) .
\end{aligned}
$$

We see that our conjecture (37), which stems from the assumption that $\lambda_{d}$ is an analytical function of $\left(J_{2}-J_{1}\right)$, is confirmed by the results of the microscopic calculations.

\section{SUMMARY}

To summarize, we have studied the properties of elementary excitations in the so-called spin nematic phase of spin-1 systems that is characterized by nontrivial quadrupole order in the absence of local spin averages. We have developed a general phenomenological theory of 
spin dynamics and relaxation for spin- 1 systems, which is based on the equations of motion for the eight-component real vector $\boldsymbol{n}$ uniting the magnetic (dipolar) and nematic (quadrupolar) order parameters. Our approach is well suited to emphasize the role of enhanced symmetry in the relaxation and is in spirit similar to the phenomenology describing relaxation in ferromagnets $\underline{49}$

The developed theory has been applied to the specific $S=1$ lattice model with isotropic bilinear and biquadratic exchange interactions, which is relevant for the physics of ultracold spin-1 Bose gases in optical lattices. In the space of the model parameters, there is a special point that exhibits an enhanced symmetry, namely, the usual $S U(2)$ (rotational) symmetry is enhanced to $S U(3)$, and the immediate vicinity of the $S U(3)$ point is occupied by the spin nematic phase. We show that the behavior of the leading term in the dependence of the magnon damping on wavevector $k$ changes from $k^{4}$ to $k^{2}$ as one moves away from the $S U(3)$ point and the symmetry is lowered to $S U(2)$. Those predictions of the phenomenological theory are consistent with the results of microscopic calculations. We also show that breaking the $S U(3)$ symmetry leads to the appearance of a homogeneous $(k \rightarrow 0)$ relaxation in the diffusive (nonpropagating) quadrupolar mode that describes fluctuations of $\left\langle S_{z}^{2}\right\rangle$.

\section{ACKNOWLEDGMENTS}

This work has been partly supported by the State Program "Nanotechnologies and Nanomaterials" of the Government of Ukraine, Project 1.1.3.27, as well as by the joint Ukrainian- Russian research program via Grant No. 0113U001823 from the National Academy of Sciences and Grant No. F53.2/045 from the State Foundation for Fundamental Research of Ukraine.

\section{Appendix: $S U(3)$ algebra and properties of octet vector products}

For the sake of the reader's convenience, we list here the basic algebraic relations 52,58 between the Gell-Mann matrices $\lambda_{\alpha}$ and the structure tensors $f_{\alpha \beta \gamma}, d_{\alpha \beta \gamma}$.
The generators of the $S U(3)$ group can be chosen in the explicit matrix representation known as Gell-Mann's matrices:

$$
\begin{aligned}
& \lambda_{1}=\left(\begin{array}{lll}
0 & 1 & 0 \\
1 & 0 & 0 \\
0 & 0 & 0
\end{array}\right), \lambda_{2}=\left(\begin{array}{ccc}
0 & -i & 0 \\
i & 0 & 0 \\
0 & 0 & 0
\end{array}\right), \lambda_{3}=\left(\begin{array}{ccc}
1 & 0 & 0 \\
0 & -1 & 0 \\
0 & 0 & 0
\end{array}\right), \\
& \lambda_{4}=\left(\begin{array}{lll}
0 & 0 & 1 \\
0 & 0 & 0 \\
1 & 0 & 0
\end{array}\right), \lambda_{5}=\left(\begin{array}{ccc}
0 & 0 & -i \\
0 & 0 & 0 \\
i & 0 & 0
\end{array}\right), \lambda_{6}=\left(\begin{array}{lll}
0 & 0 & 0 \\
0 & 0 & 1 \\
0 & 1 & 0
\end{array}\right), \\
& \lambda_{7}=\left(\begin{array}{ccc}
0 & 0 & 0 \\
0 & 0 & -i \\
0 & i & 0
\end{array}\right), \lambda_{8}=\frac{1}{\sqrt{3}}\left(\begin{array}{ccc}
1 & 0 & 0 \\
0 & 1 & 0 \\
0 & 0 & -2
\end{array}\right)
\end{aligned}
$$

The algebraic properties of Gell-Mann's matrices are given by Eq. (3), with the totally antisymmetric structure tensor $f_{\alpha \beta \gamma}$ and the totally symmetric structure tensor $d_{\alpha \beta \gamma}$ defined by their nonzero components as follows:

$$
\begin{aligned}
& f_{123}=1, \quad f_{458}=f_{678}=\frac{\sqrt{3}}{2} \\
& f_{147}=f_{246}=f_{257}=f_{345}=f_{516}=f_{637}=\frac{1}{2}, \\
& d_{118}=d_{228}=d_{338}=-d_{888}=-\frac{1}{\sqrt{3}} \\
& d_{448}=d_{558}=d_{668}=d_{778}=-\frac{1}{2 \sqrt{3}} \\
& d_{146}=d_{157}=d_{256}=d_{344}=d_{355} \\
& =d_{247}=d_{366}=d_{377}=-\frac{1}{2} .
\end{aligned}
$$

With the help of the above structure constants, one can define the symmetric and antisymmetric vector products as in Eq. (4).

If one splits the full space of octet vectors into the magnetization subspace $V_{m}$ and the quadrupolar subspace $V_{\boldsymbol{d}}$, see Eq. (10), then it is straightforward to check that the vector products have the properties

$$
\begin{aligned}
& \left(\boldsymbol{d} \wedge \boldsymbol{d}^{\prime}\right) \in V_{\boldsymbol{m}}, \quad(\boldsymbol{d} \wedge \boldsymbol{m}) \in V_{\boldsymbol{d}}, \quad\left(\boldsymbol{m} \wedge \boldsymbol{m}^{\prime}\right) \in V_{\boldsymbol{m}}, \\
& \left(\boldsymbol{d} * \boldsymbol{d}^{\prime}\right) \in V_{\boldsymbol{d}}, \quad(\boldsymbol{d} * \boldsymbol{m}) \in V_{\boldsymbol{m}}, \quad\left(\boldsymbol{m} * \boldsymbol{m}^{\prime}\right) \in V_{\boldsymbol{d}}, \quad(\text { A.4 })
\end{aligned}
$$

where $\boldsymbol{m}, \boldsymbol{m}^{\prime} \in V_{\boldsymbol{m}}$ and $\boldsymbol{d}, \boldsymbol{d}^{\prime} \in V_{\boldsymbol{d}}$.
1 A. I. Akhiezer, V. G. Bar'yakhtar, and S. V. Peletminskii, Spin Waves, (North-Holland, Amsterdam, 1968).

2 E. A. Turov, A. V. Kolchanov, V. V. Menshenin, I. F. Mirsaev, and V. V. Nikolaev, Symmetry and the Physical Properties of Antiferromagnets [in Russian], (Fizmatlit, Moscow, 2001).

3 A. M. Kosevich, B. A. Ivanov, A. S. Kovalev, Nonlinear Magnetization Waves. Dynamical and Topological Solitons (in Russian), (Naukova Dumka, Kiev, 1983); A. M. Kosevich, B. A. Ivanov, and A. S. Kovalev, Physica D 3, 363
(1981); A. M. Kosevich, B. A. Ivanov, and A. S. Kovalev, Phys. Rep. 194, 117 (1990).

4 A. F. Andreev and I. A. Grishchuck, Sov. Phys. JETP 60, 267 (1984) [Zh. Eksp. Teor. Fiz. 87, 467 (1984)].

5 N. Papanicolaou, Nucl. Phys. B 305, 367 (1988).

6 A. V. Chubukov, J. Phys.: Condens. Matter 2, 1593 (1990).

7 A. V. Chubukov, Phys. Rev. B 43, 3337 (1991).

8 N. Shannon, T. Momoi, and P. Sindzingre, Phys. Rev. Lett. 96, 027213 (2006). 
9 T. Vekua, A. Honecker, H.-J. Mikeska, and F. HeidrichMeisner, Phys. Rev. B 76, 174420 (2007).

10 T. Hikihara, L. Kecke, T. Momoi, and A. Furusaki, Phys. Rev. B 78, 144404 (2008).

11 J. Sudan, A. Luscher, A. M. Läuchli, Phys. Rev. B 80, 140402(R) (2009).

12 V. M. Loktev and V. S. Ostrovskii, Low Temp. Phys. 20, 775 (1994).

13 G. Fáth and J. Sólyom, Phys. Rev. B 51, 3620 (1995).

14 N. A. Mikushina and A. S. Moskvin, Phys. Letters A 302, 8 (2002).

15 B. A. Ivanov and A. K. Kolezhuk, Phys. Rev. B 68, 052401 (2003).

${ }^{16}$ K. Buchta, G. Fáth, Ö. Legeza, and J. Sólyom, Phys. Rev. B 72, 054433 (2005).

17 L. E. Sadler et al., Nature 443, 312 (2006).

18 S. Mukerjee, C. Xu, and J. E. Moore, PRL 97, 120406 (2006).

19 T. Grover and T. Senthil, Phys. Rev. Lett. 98, 247202 (2007).

${ }^{20}$ K. Harada, N. Kawashima, and M. Troyer, J. Phys. Soc. Jpn. 76, 013703 (2007).

21 V. I. Butrim, B. A. Ivanov, A. S. Kuznetsov, and R. S. Khymyn, Low Temp. Phys. 34, 997 (2008) [Fiz. Nizk. Temp. 34, 1266 (2008)].

${ }^{22}$ V. I. Butrim, B. A. Ivanov, and A. S. Kuznetsov, JETP Lett. 92, 151 (2010) [Pisma Zh. Eksp. Teor. Fiz. 92, 172 (2010)].

23 J.Bernatska, P. Holod, J. Phys. A: Math. Gen. 42, 075401 (2009).

24 T. A. Toth, A. M. Läuchli, F. Mila, and K. Penc, Phys. Rev. B 85, 140403(R) (2012)

${ }^{25}$ K. Rodriguez, A. Argüelles, A. K. Kolezhuk, L. Santos, and T. Vekua, Phys. Rev. Lett. 106, 105302 (2011).

26 G. De Chiara, M. Lewenstein, and A. Sanpera, Phys. Rev. B 84, 054451 (2011)

27 M. Yu. Kovalevskii, Theor. Math. Physics 168, 1064 (2011) [Teor. Mat. Fizika, 168, 245 (2011)].

28 See Y. Kawaguchi and M. Ueda, Phys. Reports 520, 253 (2012), and references therein.

29 S. Trotzky, P. Cheinet, S. Fölling, M. Feld, U. Schnorrberger, A. M. Rey, A. Polkovnikov, E. A. Demler, M. D. Lukin, and I. Bloch, Science 319, 295 (2008).

30 Y.-J. Lin, R. L. Compton, K. Jiménez-García, J. V. Porto, and I. B. Spielman, Nature 462, 628 (2009).

${ }^{31}$ K. Kim, M.-S. Chang, S. Korenblit, R. Islam, E. E. Edwards, J. K. Freericks, G.-D. Lin, L.-M. Duan, and C. Monroe, Nature 465, 590 (2010).

32 R. Jördens, L. Tarruell, D. Greif, T. Uehlinger, N. Strohmaier, H. Moritz, T. Esslinger, L. De Leo, C. Kollath, A. Georges, V. Scarola, L. Pollet, E. Burovski,
E. Kozik, and M. Troyer, Phys. Rev. Lett. 104, 180401 (2010).

33 X.-S. Ma, B. Dakic, W. Naylor, A. Zeilinger, P. Walther, Nature Physics 7, 399 (2011).

34 J. Simon et al., Nature 472, 307 (2011).

35 J. Struck, C. Ölschläger, R. Le Targat, P. Soltan-Panahi, A. Eckardt, M. Lewenstein, P. Windpassinger, K. Sengstock, Science 333, 996 (2011).

36 T.-L. Ho, Phys. Rev. Lett. 81, 742 (1998).

37 T. Ohmi and K. Machida, J. Phys. Soc. Jpn. 67, 1822 (1998).

38 M. D. Barrett, J. A. Sauer, and M. S. Chapman, Phys. Rev. Lett. 87, 010404 (2001).

39 J. Stenger, D. M. Stamper-Kurn, H. J. Miesner, A. P. Chikkatur, and W. Ketterle, Nature (London) 396, 345 (1998).

40 E. Demler and F. Zhou, Phys. Rev. Lett. 88, 163001 (2002).

41 S. K. Yip, Phys. Rev. Lett. 90, 250402 (2003).

42 F. Zhou and M. Snoek, Ann. Phys. 308, 692 (2003).

43 A. Imambekov, M. Lukin, and E. Demler, Phys. Rev. A 68, 063602 (2003).

44 A. Imambekov, M. Lukin, and E. Demler, Phys. Rev. Lett. 93, 120405 (2004).

45 M. Snoek and F. Zhou, Phys. Rev. B 69, 094410 (2004).

46 M. Rizzi, D. Rossini, G. De Chiara, S. Montangero, and R. Fazio, Phys. Rev. Lett. 95, 240404 (2005).

47 M.-C. Chung and S. Yip, Phys. Rev. A 80, 053615 (2009).

${ }^{48}$ C. D. Batista, G. Ortiz, and J. E. Gubernatis, Phys. Rev. B 65, 180402(R) (2002).

49 V. G. Bar'yakhtar, Sov. Phys. JETP 60, 863 (1984) [ Zh. Eksp. Teor. Fiz. 87, 1501 (1984)].

50 E. Ercolessi, G. Marmo, G. Morandi, and N. Mukunda, Int. J. Mod. Phys. A 16, 5007 (2001).

51 B. A. Ivanov, R. S. Khymyn, and A. K. Kolezhuk, Phys. Rev. Lett. 100, 047203 (2008).

52 A. J. Macfarlane, A. Sudbery, and P. H. Weisz, Commun. Math. Phys. 11, 77 (1968).

53 Akira Onuki, Phase transition dynamics (Cambridge University Press, 2004).

54 A. K. Kolezhuk, Phys. Rev. B 78, 144428 (2008).

55 K. Harada and N. Kawashima, Phys. Rev. B 65, 052403 (2002).

56 B. A. Ivanov, A. Yu. Galkin, R. S. Khymyn, and A. Yu. Merkulov, Phys. Rev. B 77, 064402 (2008).

57 M. I. Kaganov and V. M. Tsukernik, Zh. Eksp. Teor. Fiz. 34, 1610 (1958) [Sov. Phys. JETP 7, 1107 (1958)];

58 M. Gell-Mann, The Eightfold way, Caltech Report CTSL20 (1961), unpublished; reproduced in: The Eightfold Way, M. Gell-Mann and Y. Ne'eman (Benjamin Inc., New York 1964). 